\title{
Revisiting Cross-channel Information Transfer for Chromatic Aberration Correction
}

\author{
Tiancheng Sun ${ }^{2,1}$ Yifan Peng ${ }^{3,1}$ Wolfgang Heidrich ${ }^{1,3}$ \\ ${ }^{1}$ King Abdullah University of Science and Technology, Thuwal, Saudi Arabia \\ ${ }^{2}$ IIIS, Tsinghua University, Beijing, China \\ ${ }^{3}$ The University of British Columbia, Vancouver, Canada
}

\begin{abstract}
Image aberrations can cause severe degradation in image quality for consumer-level cameras, especially under the current tendency to reduce the complexity of lens designs in order to shrink the overall size of modules. In simplified optical designs, chromatic aberration can be one of the most significant causes for degraded image quality, and it can be quite difficult to remove in post-processing, since it results in strong blurs in at least some of the color channels. In this work, we revisit the pixel-wise similarity between different color channels of the image and accordingly propose a novel algorithm for correcting chromatic aberration based on this cross-channel correlation. In contrast to recent weak prior-based models, ours uses strong pixelwise fitting and transfer, which lead to significant quality improvements for large chromatic aberrations. Experimental results on both synthetic and real world images captured by different optical systems demonstrate that the chromatic aberration can be significantly reduced using our approach.
\end{abstract}

\section{Introduction}

Modern optical systems usually consist of dozens of heavy and complex lenses, which are used to compensate for aberrations of all kinds [5]. However, the increase of the computing power and the demand for more compact devices, has recently started a push to optical designs with reduced complexity, that instead use computational imaging methods to recover high quality photographs [23, 7, 18, 19]. This is of significance particularly in mobile devices, where form factor constraints mandate optical designs with a small number of lens elements, and the small size of components complicates manufacture and alignment.

Images captured by uncorrected lenses may suffer from both monochromatic aberrations (e.g. spherical, astigmatism, coma, field curvature), as well as chromatic aberrations. Further, in the absence of optical image stabilizers (OIS), the captured images may be subject to motion blur.
With optical means, chromatic aberration can only be corrected by involving multiple lens elements with different geometric shapes and different refractive indices. Thus, there is a high demand on an effective deblurring approaches specially targeting the reconstruction of color images captured by reduced complexity optics, which is also the objective of our work. Specifically, we tackle this problem by exploiting the pixel correlation between color channels. In this way, we reduce the problem to single channel deblurring, and show that high-quality color images can be recovered with cross-channel information.

\subsection{Related work}

In the following we will summarize two types of related work: general deconvolution/deblurring research, and work that specifically targets chromatic aberrations.

Image deblurring Early development of deconvolution algorithms include direct frequency inverse, Wiener filter [29], which fail to tackle those frequencies that are zeroed out by the blur kernel, and thus may result in ringing artifacts. Later development of iterative algorithms [15] extracts better results but may amplify noise level.

In recent years, researchers have attempted to add extra prior knowledge into deconvolution to restore highfrequency information. Chan et al. [2] used a total variation prior, where a global optimum can be obtained with convex optimization. Alternative non-convex regularization terms have been investigated [12, 10], empirically giving improved results at reasonable local optimum. Cho et al. [3] explored to match gradient distribution in order to restore mid-frequency information. All these methods rely on weak statistic priors for image restoration.

General image deconvolution model requires a known convolution kernel, which could be impractical to calibrate, for instance motion blur. Accordingly, blind deconvolution algorithms have been extensively studied aiming to reconstruct the latent image and estimate the kernel simultaneously [24]. Similar to their non-blind counterparts, a wide 
variety of priors are introduced to resolve reasonable results in image enhancement [11, 13, 26]. An investigation on the use of sparse gradient priors for blind deconvolution via total variation can be found in [20].

Chromatic aberration correction The chromatic aberration observed in images are caused by the wavelengthdependence of focal length. Different point spread functions (PSFs) in channels result in color fringes on sharp edges. Alternative low-level technique has been applied to remove the color fringes in conventional complex optical systems [9, 4]. However, these methods fail to tackle the large aberration induced by uncorrected lenses.

Schuler et al. [22] first proposed an aberration removal algorithm for a single lens in YUV color space. Further, a convex cross-channel prior is developed and efficiently solved in [7]. Accordingly, several simplified optical applications have been presented - a low-end camera lens is corrected via optical computing in [31]; DOEs are optimized along with aberration removal algorithm to resolve high-quality images [18, 19].

Although challenging, it is practically of significance to remove aberrations without the knowledge of kernels. Besides simply implementing blind deconvolution on each channel, the symmetry of the convolution kernel are exploited in [23], and the geometric and visual priors are investigated in [30]. While existing models are successful for yielding reasonably good results with chromatic aberration mitigated, they may over-simplify the drastic spatiallyvarying defocus effect (e.g. commonly at the borderline of sharp foreground and blurred background, resulting in a failure of geometric symmetry assumption). These methods may result in color fidelity loss of resolved images (as we will discuss in our experiments).

\subsection{Motivation and contribution}

We base our work on two primary observations. First, as shown in Fig. 1, the dependence of focal length on wavelength results in severe chromatic aberration, which is often too challenging to robustly recovery with state-of-theart gradient transfer schemes. However, at least one of the channels usually exhibits relatively sharper focus (and could be recovered more easily) when the camera has been focused at a spatial point. Second, despite the different pixel intensity between channels, the high-level structures conveyed by the channels are mostly identical. These facts have inspired us to exploit essential similarity of a color image, and accordingly transfer pixel information across channels, to blindly remove the severe chromatic aberration introduced by uncorrected or complexity reduced optics. In particular, the technical contributions include:

- We investigate the cross-channel content similarity of natural images by deriving a novel image formation
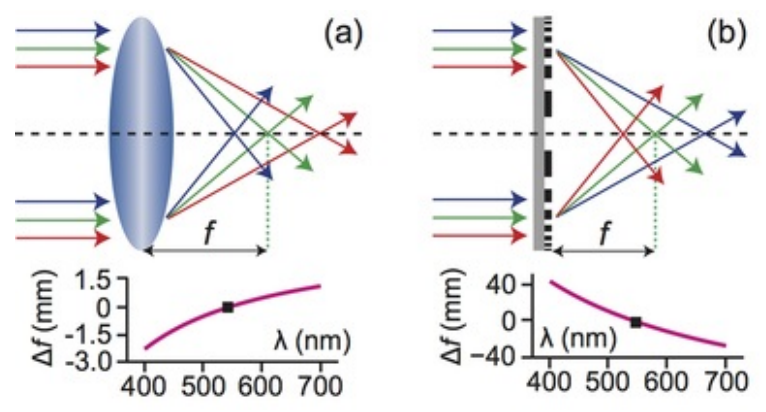

Figure 1: Relation between focal length and wavelength in two typical optical systems. The focal length of a refractive lens increases when the wavelength increases (left); while that of a diffractive lens exhibits the opposite way (right). The color disperson of a diffractive lens is much larger than that of a refractive lens. (Image credit at [27])

model to express one channel using another channel. Instead of relying on weak statistical prior knowledge, our method uses a strong pixel-wise correlation, leading to a deconvolution that can tackle considerable chromatic aberration efficiently.

- We compare our algorithm against state-of-the-art methods, and ours exhibits overall better performance in terms of removing chromatic aberration while suppressing edging artifacts. We also work in a blind deconvolution mode without previously known kernels.

- We verify our method on data captured by simple refractive lens and diffractive lens, suggesting its universality and robustness across diverse optical systems, making our method a promising candidate for developing lightweight computational imaging solutions.

\section{Image formation model}

Cross-channel similarity. To revisit the problem of transferring information from a sharper channel to others, we start by exploring the cross-channel similarity using natural images with no (or only imperceptible) aberration. Different channels in natural images convey largely redundant information, since strong edges and textures tend to be aligned between the channels. However, although the correlation between channels is strong due to joint structure [14] in edge and texture features, the exact nature of this correlation can be complicated and difficult to express in closed form. Recent works have attempted to model specific forms of channel correlation, such as sparse hue changes at object boundaries. However, there exist a myriad of other types of hue changes in natural images, such as color gradients, rainbows etc., thus resulting in relationship that is in general highly non-linear.

In our work, we follow the strategy of Taylor expansion to mode this inter-channel dependency. Although it is hard 

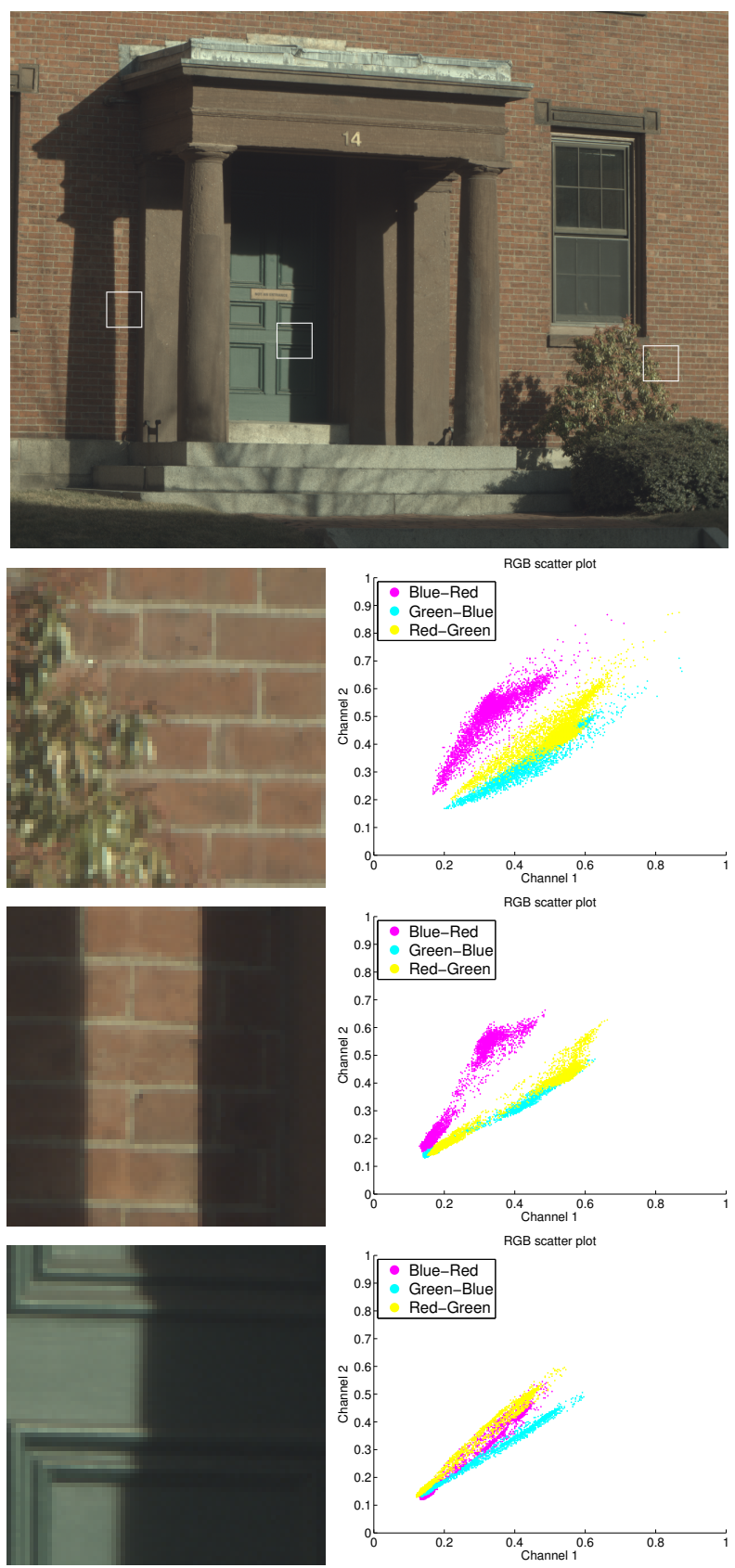

Figure 2: The scatter plots of pixel values from three patches selected from a sample image. The red, yellow, blue plots correspond to RG plot, GB plot, and BR plot of each patch. The linearity within small patches can been clearly observed (distributions of color plots).

to formulate the correlation from a global view, one can still linearize the problem within small windows cropped from an image. Specifically, we reasonably assume under a small window patch which contains only few features (e.g. edges, corner points, etc.), the pixel intensity of one channel $\mathbf{I}_{t}$ can be expressed as a weighted sum of higher-order derivatives

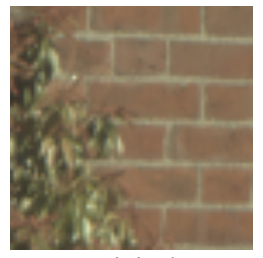

original

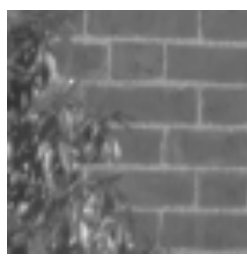

original green

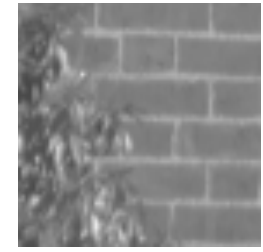

reconstruct red

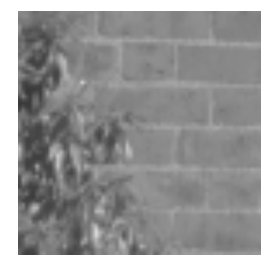

original red

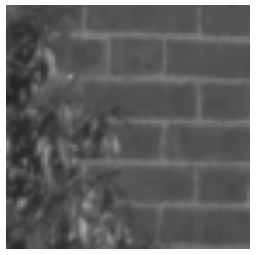

reconstruct blue

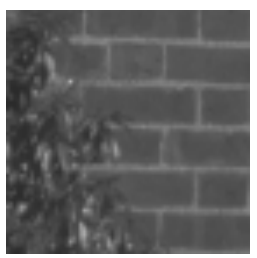

original blue
Figure 3: Results of our similarity model tested on a small patch cropped from the image by solving Eq. 2 The red and blue channels are expressed sparsely using the pixel intensities of green channel. Notice that the reconstructed windows (upper middle and upper right) are almost identical to the original ones (lower middle and lower right), indicating the effectiveness of our cross-channel similarity model.

from another channel $\mathbf{I}_{\mathrm{s}}$, similar to a Taylor expansion:

$$
\begin{aligned}
\mathbf{I}_{\mathrm{t}} & \approx \alpha_{0} \cdot \mathbf{1}+\alpha_{1} \cdot \mathbf{I}_{\mathrm{s}}+\alpha_{2} \cdot \nabla_{\mathrm{x}} \mathbf{I}_{\mathrm{s}}+\alpha_{3} \cdot \nabla_{\mathrm{y}} \mathbf{I}_{\mathrm{s}}+\cdots \\
& =\mathbf{T}\left(\mathbf{I}_{\mathrm{s}}\right) \cdot \boldsymbol{\alpha},
\end{aligned}
$$

where

$$
\mathbf{T}\left(\mathbf{I}_{\mathrm{s}}\right)=\left[\begin{array}{lllll}
\mathbf{1} & \mathbf{I}_{\mathrm{s}} & \nabla_{\mathrm{x}} \mathbf{I}_{\mathrm{s}} & \nabla_{\mathrm{y}} \mathbf{I}_{\mathrm{s}} & \cdots
\end{array}\right],
$$

and $\boldsymbol{\alpha}$ are the coefficients to be fitted. Notice that this is the model for an intrinsic (i.e. aberration free) image.

The proposed model is validated on selected images from dataset in [1]. As shown in Fig. 2, we crop three patches from the original image, and plot them out subject to their RGB values. From the patches' graphs, we can observe noticeable linearity which is useful in correcting chromatic aberration.

Then, the problem (Eq. 11) can be treated as a fitting operation with its least-square solution being calculated efficiently using pseudo-inverse, to find $\boldsymbol{\alpha}$ by solving:

$$
\boldsymbol{\alpha}_{\mathrm{opt}}=\underset{\boldsymbol{\alpha}}{\operatorname{argmin}}\left\|\mathbf{I}_{\mathrm{t}}-\mathbf{T}\left(\mathbf{I}_{\mathrm{s}}\right) \cdot \boldsymbol{\alpha}\right\|_{2}^{2} .
$$

In practice, we only expand the series to the second order derivative of the image (i.e. $\nabla_{\mathrm{xx}} \mathbf{I}, \nabla_{\mathrm{xy}} \mathbf{I}$ and $\nabla_{\mathrm{yy}} \mathbf{I}$ ). The reconstructed result of a small window patch by solving Eq.2 is shown in Fig. 3. We see that the red and blue channels are reasonably expressed using the pixel intensities of green channel, exhibiting very similar content to the original ones.

Chromatic aberration formation. The chromatic aberrations in the captured images are mainly caused by different focusing contributions in different channels. Generally, 
we denote $\mathbf{I}_{\mathrm{s}}, \mathbf{J}_{\mathrm{s}}, \mathbf{N}_{\mathrm{s}}$ as the underlying sharp image, observed image, and additive noise in channel $s$, respectively. The observed image in channel $s$ can be formatted as:

$$
\mathbf{J}_{\mathrm{s}}=\mathbf{B}_{\mathrm{s}} * \mathbf{I}_{\mathrm{s}}+\mathbf{N}_{\mathrm{s}},
$$

where $\mathbf{B}_{\mathrm{s}}$ is the point spread function (PSF) of channel $s$. Although the contents in $\mathbf{I}_{\mathrm{S}}$ are strongly correlated across different channels, the PSFs $\left(\mathbf{B}_{\mathrm{s}}\right)$ may vary drastically between channels, which overall leads to the chromatic aberration such as red or purple fringes.

\section{Image reconstruction}

We rely on the similarity model from above to resolve the chromatically aberrated images captured by complexity reduced lenses. We first resolve a sharp reference channel using a mature deconvolution scheme, then develop a method for individual image patches where we assume a constant PSF as well as the validity of our cross-channel model derived from Eq.2 After that, we incorporate this patch-based solution into an efficient algorithm at full image scale.

Reference channel deblurring. Given a blurry color image, there is at least one channel exhibiting relatively sharper information, which is usually the green channel 17 6]. For generality, we denote the relatively sharper channel as $\mathbf{I}_{\text {ref. }}$. We then apply a blind deconvolution algorithm on this channel, in order to suppress the monochromatic aberrations and possibly the motion blur. Section 4 details this single-channel deblurring algorithm.

Cross-channel information transfer. Given a clear image of reference channel $\mathbf{I}_{\text {ref }}$, we build our algorithm to deblur other channels by transferring the sharp information across channels. By introducing the blur kernel into our similarity model, we now formulate an optimization problem to deconvolve small image patches, as follows:

$$
\begin{aligned}
\min _{\boldsymbol{\alpha}, \mathbf{B}_{\mathrm{s}}}\left\|\mathbf{J}_{\mathrm{s}}-\mathbf{B}_{\mathrm{s}} *\left(\mathbf{T}\left(\mathbf{I}_{\mathrm{ref}}\right) \cdot \boldsymbol{\alpha}\right)\right\|_{2}^{2}+ \\
\mu\left\|\mathbf{B}_{\mathrm{s}}\right\|_{2}^{2}+\nu \sum_{\mathrm{a}}\left\|\nabla_{\mathrm{a}} \mathbf{B}_{\mathrm{s}}\right\|_{2}^{2},
\end{aligned}
$$

where $\mathbf{I}_{\text {ref }}$ is the image patch of the sharp reference channel and, $\mathbf{J}_{\mathrm{s}}$ is that of the blurred channel $s$. The classical $l_{2}$ fusion data term and gradient priors are added to regularize PSFs. Notice that the above problem is biconvex and can be solved by alternatively updating $\boldsymbol{\alpha}$ and $\mathbf{B}_{\mathrm{s}}$. The $\mathbf{B}_{\mathrm{s}}$ step (PSF estimation step) can be solved efficiently in Fourier domain, and the $\boldsymbol{\alpha}$ step (cross channel transfer step, aka. CCT step) can be solved by using pseudo-inverse. Compared to previous work [7, 30], we don't add any statistical prior to the CCT term, but use a set of appropriate bases (i.e. $\mathbf{T}\left(\mathbf{I}_{\mathrm{ref}}\right)$ ) which are derived from other channels to restore the blurred image. In other words, we mainly rely on
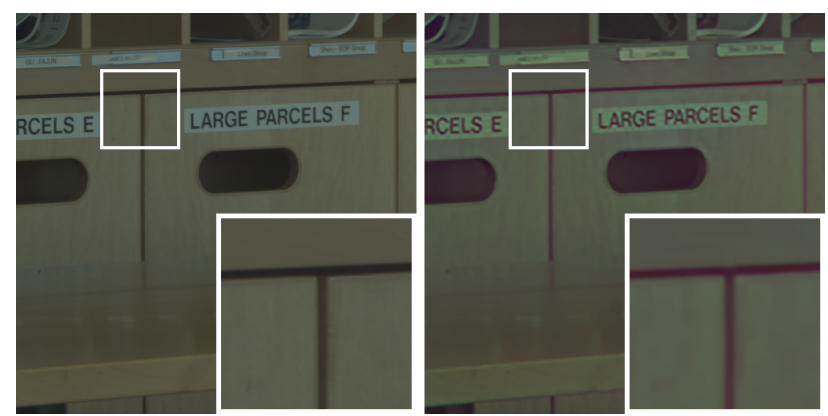

Figure 4: Left: ground truth image; right: recovered image using 0-order CCT initialization. Notice that in the zoom-in insets the black lines have turn red.

other sharp channels rather than severely blurred channel itself to extract effective information. Thus, more accurate pixel information can be transferred with a simpler model.

Notice that there is a trade-off between the patch sizes for PSF estimation and the cross-channel model. On the one hand, the window size for PSF estimation should be as large as possible, for the PSF to be recovered robustly. On the other hand, the approximation of one color channel by lower-order terms of another channel works best in relatively small tiles. We resolve this trade-off by working at two different scales - smaller patches (CCT windows) for the color model and larger window for PSF estimation. We discuss these and other parameters in Section 4.

Initial estimation. Since the alternating strategy can only converge to local optimum, a good initial estimation of sharp image is of significance to speed up the convergence and avoid noticeable artifacts. An intuitive solution for initial guess is the 0 -order CCT:

$$
\min _{\boldsymbol{\alpha}}\left\|\mathbf{J}_{\mathrm{s}}-\left(\alpha_{0}+\alpha_{1} \mathbf{I}_{\mathrm{ref}}\right)\right\|_{2}^{2} .
$$

However, using this initial guess may still result in artifacts. As shown in Fig. 4. most black lines have turned red. This is because those black lines or sharp peaks have been smoothed away, especially in red and blue channels where the blur kernels are of non-trivial size. Thus, simply implementing CCT may over-fit the smoothed signal, as shown in Fig. 5

To fix this issue, we seek to directly shift the signal from reference channel to other channels rather than data fitting, indicated as follows:

$$
\mathbf{I}_{\mathrm{s}}=\frac{\operatorname{mean}\left(\mathbf{J}_{\mathrm{s}}\right)}{\operatorname{mean}\left(\mathbf{I}_{\mathrm{ref}}\right)} \cdot \mathbf{I}_{\mathrm{ref}} .
$$

where $\mathbf{I}_{\mathrm{s}}$ is the sharp image patch of channel $s$ to be estimated, and $\mathbf{J}_{\mathrm{s}}$ is the corresponding observed image patch. By applying Eq. 6 in the initialization, the red line artifact can be drastically mitigated, improving the conditioning for 


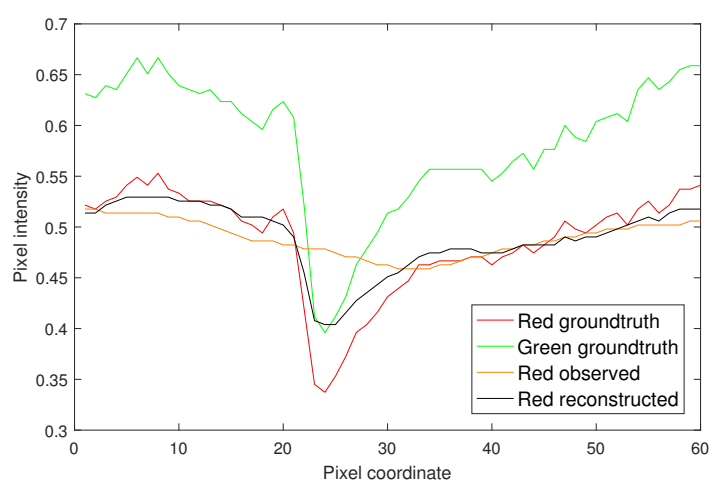

Figure 5: Pixel value plot of a 1-d slice of the image. The distributions of the original red and green signal are quite similar, while the intensity peak in the signal is smoothed out in the observed red channel (orange plot). Thus, simply relying on CCT can only reconstruct a small peak, which leads to the noticeable red line artifacts.

further deblurring. Please refer to Fig. 8 in the next section.

Patch blending. Possible methods to merge these small window patches include overlapping the small windows and extracting the mean or median of each pixel value, or just extracting the value of the nearest window. Marwah et al. [17] have shown that the median-based method outperforms other alternatives. However, the images reconstructed by the state-of-the-art suffer from vertical or horizontal edging artifacts. Thus, we multiply a Hamming window before taking the average. This strategy leads to less edging artifact appearing and recovers a higher image quality.

Joint algorithm. The algorithm for correcting the chromatic aberration is summarized in Alg.11. Given a corrupted color image, we firstly apply an efficient blind deconvolution on the less blurred channel image (usually the green one). Next, relying on the deconvolved channel image, we implement PSF estimation and CCT alternatively on images from other channels. Here in the algorithm, we use $\mathbf{I}, \mathbf{J}$ to denote the full-size image, and $\mathbf{I}^{\mathrm{PSF}} / \mathbf{I}^{\mathrm{CCT}}, \mathbf{J}^{\mathrm{PSF}} / \mathbf{J}^{\mathrm{CCT}}$ to denote the patch images from PSF/CCT estimation windows.

\section{Implementation and discussion}

In this section, we present selected experimental results to verify the effectiveness of our algorithm. Refer to supplementary document for full resolution results.

\subsection{Parameters and analysis}

Blind deconvolution pre-processing. We use the algorithm in [11] as the first step processing to deconvolve the reference channel. The applied algorithm reduces achromatic aberration of most kinds, and more importantly al-

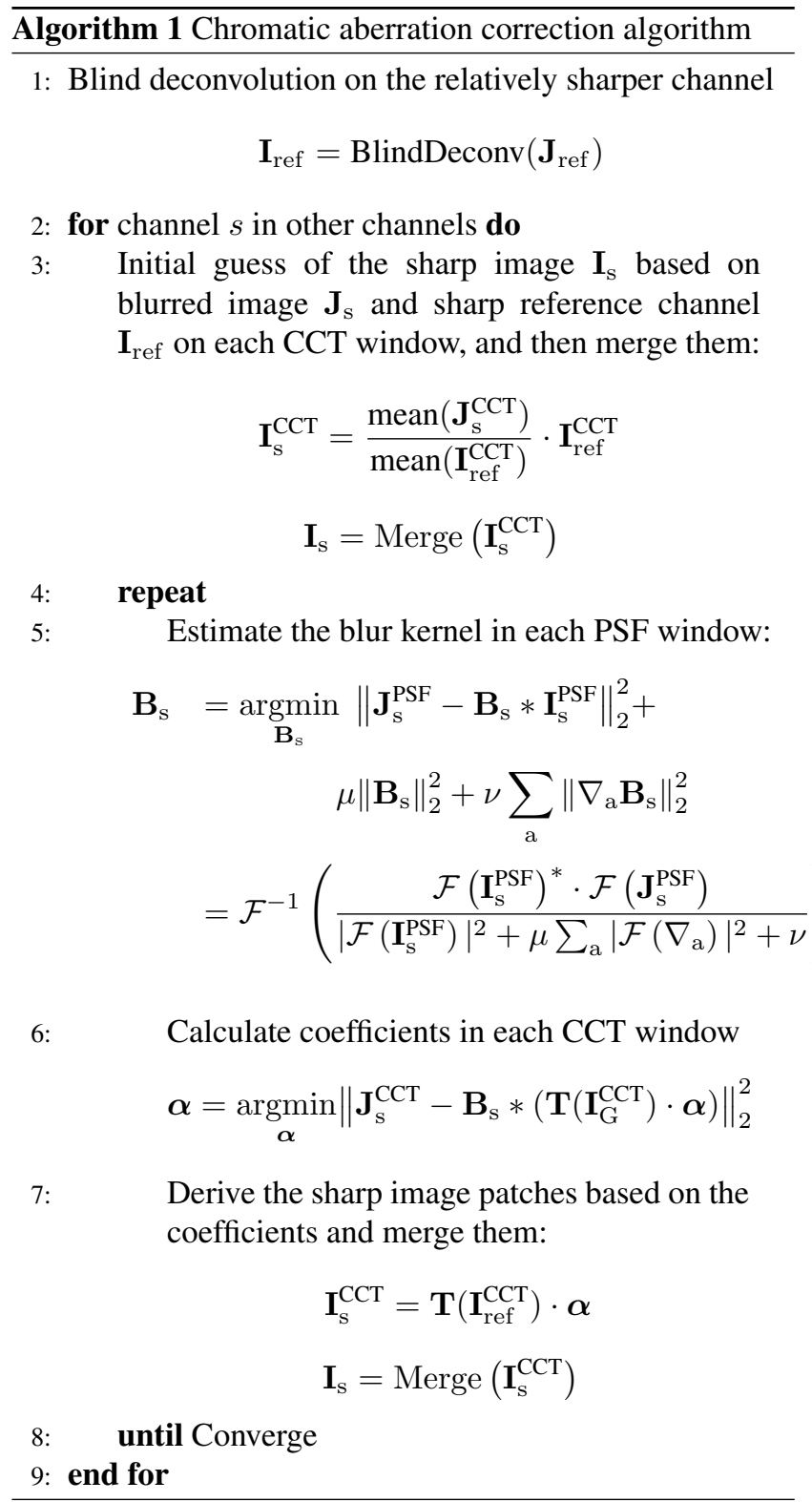

lows to control the "aggressiveness" of blind deconvolution so as to suppress artifacts that may affect the restored image quality of other channels.

PSF estimation window size. Intuitively, the window size of PSF estimation should be set large to contain sufficient features in order to yield a good estimation. Practically, PSF distribution may vary drastically due to the spatial variance of the scene. Empirically, this window size is set between $33 \%$ to $50 \%$ of image size. In order to avoid edging artifacts, both CCT windows and PSF estimation windows should overlap, which we empirically set to $1 / 5$ of the window size. We set the two regularizer parameters in estimating $\operatorname{PSF}(\mu$ and $\nu$ ) to 0.3 in the followings. 


\begin{tabular}{|c|c|c|c|c|c|c|c|c|c|}
\hline & & 30 & 40 & 50 & 60 & 70 & 80 & 90 & 100 \\
\hline & 500 & 33.46 & 34.23 & 34.64 & 34.94 & 35.00 & 35.09 & 35.27 & 35.30 \\
\hline $\mathrm{R}$ & 600 & 33.52 & 34.20 & 34.50 & 34.76 & 34.96 & 34.82 & 34.78 & 34.77 \\
\hline e & 700 & 33.51 & 34.40 & 34.84 & 34.97 & 34.87 & 34.72 & 34.90 & 34.76 \\
\hline d & 800 & 33.70 & 34.49 & 34.83 & 34.92 & 34.87 & 34.69 & 34.50 & 34.45 \\
\hline & 900 & 34.14 & 34.70 & 34.68 & 34.88 & 34.91 & 34.92 & 34.96 & 34.78 \\
\hline & 1000 & 34.08 & 34.55 & 34.71 & 34.86 & 34.62 & 34.46 & 34.18 & 33.60 \\
\hline & 500 & 35.64 & 36.09 & 36.28 & 36.38 & 36.59 & 36.74 & 36.80 & 36.80 \\
\hline B & 600 & 35.25 & 35.82 & 36.04 & 36.30 & 36.46 & 36.50 & 36.51 & 36.65 \\
\hline 1 & 700 & 35.51 & 35.86 & 36.10 & 36.23 & 36.43 & 36.44 & 36.51 & 36.70 \\
\hline $\mathrm{u}$ & 800 & 35.65 & 36.03 & 36.39 & 36.45 & 36.41 & 36.67 & 36.81 & 36.78 \\
\hline e & 900 & 36.08 & 36.52 & 36.78 & 36.77 & 36.82 & 36.80 & 36.80 & 36.79 \\
\hline & 1000 & 36.28 & 36.60 & 36.76 & 36.78 & 36.78 & 36.73 & 36.69 & 36.74 \\
\hline
\end{tabular}

Figure 6: PSNR heatmap of red and blue channels of a restored image (Figure 8) subject to different parameter settings. The horizontal axis denotes CCT window size while the vertical one denotes PSF estimation window size.

Cross-channel transfer window size. Conceptually, the CCT window should contain few features in order to exploit the content linearity. In practice, we also observe that an overly large CCT window may result in a pale or low color fidelity image. It can't be too small as well, otherwise, the content of the small window patch may fail to contain sufficient color information subject to the full spectrum, making the problem strongly ill-posed. For an image with $1,400 \times 1,000$ pixels, the size of CCT window is set $70 \times 70$ pixels.

Robustness. The sizes of PSF estimation window and CCT window can be estimated from the size of image. To assess the robustness subject to different parameters, we run our algorithm on the upper image of Fig. 8 with different settings of PSF and CCT windows (Fig. 6). We found that the image quality doesn't change much when window sizes change. The optimal PSNRs lie on a plateau (see the red color blocks) rather than on individual sharp peaks, suggesting our algorithm does not need to fine-tune many parameters.

Convergence. Although the stopping criteria in Alg. 1 is not deterministic, the alternating optimization converges very quickly in practice. Empirically, there is little perceptible change after the first iteration, and the similarity metrics continue to improve for a few iterations. Thus we fix this parameter to 3 iterations.

Efficiency. Denote $N, N_{\mathrm{p}}, N_{\mathrm{c}}$ as the size of full image, PSF estimation window, and CCT window, respectively. The time complexity for a single iteration can be indicated as $O\left(\left(N-N_{\mathrm{p}}\right)^{2} \cdot \log N_{\mathrm{p}}^{2}+\left(N^{2}+N^{3} / N_{\mathrm{c}}^{3}\right)\right)$. Notice that the first term is almost the same as that of [6]. Without a convergence problem when restoring the sharp image, the second term is relatively small compared to the stateof-the-art. In terms of practical computational efficiency, the proposed algorithm restores one blurry channel image $(1,400 \times 1,000$ pixels $)$ within 7 seconds, using a Matlab
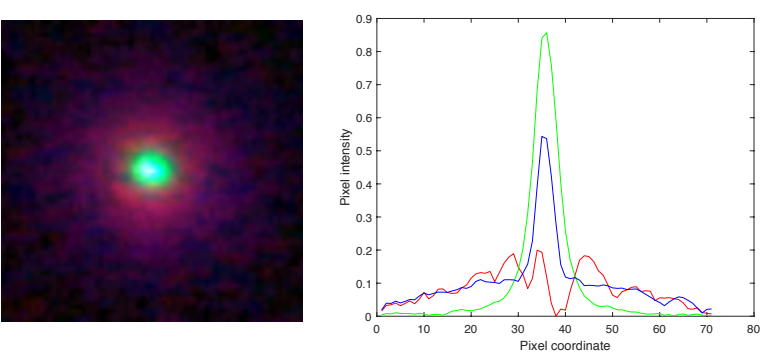

Figure 7: PSF illustration for the synthetic images (left), with its cross-section intensity in three channels (right). Notice that the green channel has the sharpest PSF distribution.

Table 1: Quantitative comparison on synthetic images, indicated as averaged PSNR and SSIM values.

\begin{tabular}{|l|l|l|}
\hline & PSNR & SSIM \\
\hline Blurred images & 30.50 & 0.8911 \\
\hline Krishnan et al. [11] & 32.65 & 0.9157 \\
\hline Heide et al. [6] & 33.43 & 0.9266 \\
\hline Ours & $\mathbf{3 4 . 7 2}$ & $\mathbf{0 . 9 4 3 4}$ \\
\hline \hline Non-blind [7] & 35.45 & 0.9426 \\
\hline
\end{tabular}

implementation on a PC with Intel Core i7 CPU at 4.0GHz. Please refer to supplementary document for detailed analysis.

\subsection{Synthetic results}

We test our algorithm on the images from dataset [1] for quantitative evaluation. All 29 synthetic images without cross-channel difference are used for evaluation. The images convolved with a PSF that is derived from a real Fresnel lens (Fig.7), whose chromatic aberration (corrupted with strong noise) is much more severe than that of conventional refractive lenses. We compare our algorithm against 2 blind methods and 1 non-blind method with averaged PSNR and SSIM [28] assessments (Tab. 1). Specifically, Krishnan's method simply applies blind deconvolution on three channels individually. Instead, Heide's methods have considered the cross-channel correlation to further improve the color fidelity. Our algorithm outperforms these blind aberration corrections. Moreover, compared with the non-blind deconvolution [7], our results are still competitive. Please refer to supplemental document for full resolution results.

Selected cropped regions are presented to highlight those detail structures in Fig. 8. Specifically, the desk scene (top row) shows that our algorithm is capable of eliminating those red fringes even though the color are scattered over a large region because of defocus. Especially, the red line artifact (bottom row) has been resolved with the aid of our initialization guess. We encourage readers to refer to the supplemental document for more results. 


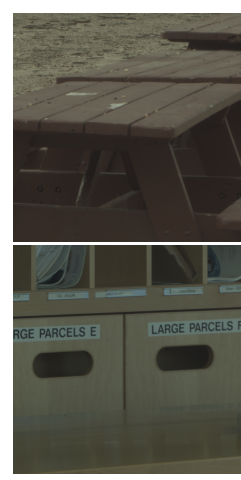

(a) Groundtruth

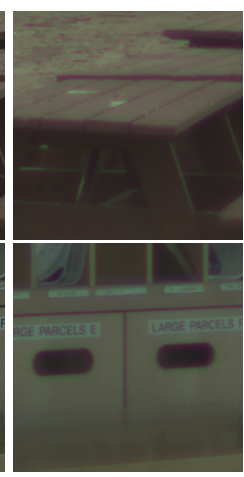

(b) Blurred

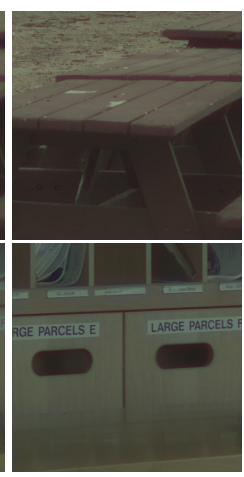

(c) Non-blind

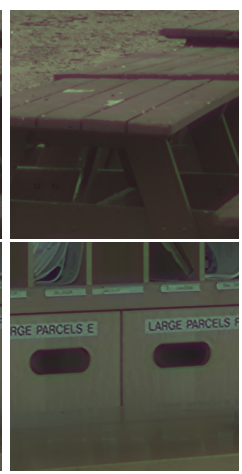

(d) Krishnan et al.

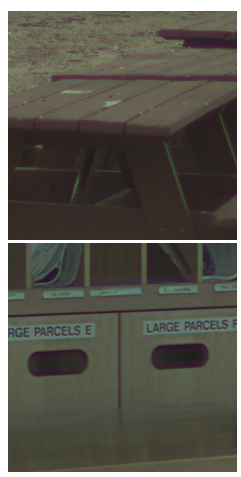

(e) Yue et al

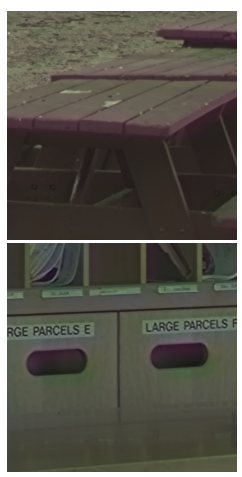

(f) Heide et al.

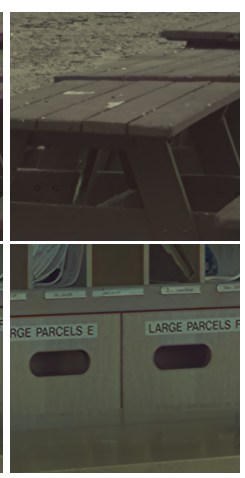

(g) Ours

Figure 8: Images recovered via applying different algorithms. Refer to the supplemental document for more results.

\subsection{Real world results}

We also apply our algorithm to the images captured using a refractive lens and a diffractive lens, respectively.

Refractive lens data. The images captured by conventional plano-convex lenses are shown in Fig. 9. In the first two columns, we compare with the work in [7], which belongs to non-blind deconvolution schemes. The comparison suggests that our algorithm removes the aberration without sacrificing fine-grain details (second column). Particularly, in the first column the color fringes have been erased even though partial image is out-of-focus. Surprisingly, our results are slightly sharper than that of the non-blind version in some cases. However, we still observe artifacts in our restored images, such as the color fidelity lost in number "16". The results in the third column come from [31], notice that the color fringe along the window and the color spots are completely removed. Although our intention wasn't dealing with motion blur, we test our algorithm on data from [11]. In the fourth column, we show that our algorithm is also capable of removing a decent amount of motion blur.

Diffractive lens data. Recently, relying on diffractive optics to shrink lens volume has been an attractive direction [25, 18]. However, the induced large color fringe still needs to be corrected. The images captured by a diffractive Fresnel lens [6] are evaluated (Fig. 10]. From the second and fourth column, we see that large chromatic fringes are eliminated correctly by our algorithm. As well, even for the color fringes on high reflection components (first column) and large hue shifts (rubic cube in third column), our algorithm is sufficiently robust to correct them properly.

\subsection{Discussion}

In our algorithm we don't add additional gradient prior on latent image as regularizer when implementing the nonblind deconvolution part. We empirically found that the image quality doesn't improve sharply with adding a cross- channel gradient prior like in [7]. This suggests that our strong pixel-wise transfer in data fitting term has fully contained the information provided by the weak statistical priors. Refer to the supplementary document for details.

Although high image quality has been achieved in our implementation, small artifacts remain. In those scenarios where none of the channel images is sufficiently sharp, ringing artifacts could be introduced during the deconvolution process of the reference channel and spread from there into other channels. This may result in achromatic ringing, which is visually less noticeable than chromatic ringing, but still worth investigating more. Also, we approximate the cross-channel correlation via a simple math scheme (Eq. 1) and move it into the data fitting term, which shall be computationally efficient but may not be very precise for all natural scenes. One possible improvement is to apply learningbased strategies to exploit a more comprehensive model.

\section{Conclusion}

We propose a post-processing algorithm to correct chromatic aberration for color images and demonstrate best-inclass performance on removing such chromatic aberration without calibrating optical systems. We have validated the robustness of our algorithm with a variety of synthesized images, where we obtain competitive results as state-of-theart methods. The implementations on real world images captured by two different complexity reduced lenses have further verified the effectiveness and the universal.

We envision this method be an effective tool to enhance image quality especially in scenarios where high-quality optics are not feasible due to weight or form-factor considerations, such as in mobile devices. Importantly, our algorithm is capable of resolving images captured by a wide range of optical systems, including diffractive optics which exhibits severe chromatic aberration where most of state-ofthe-art chromatic aberration correction methods fail. 


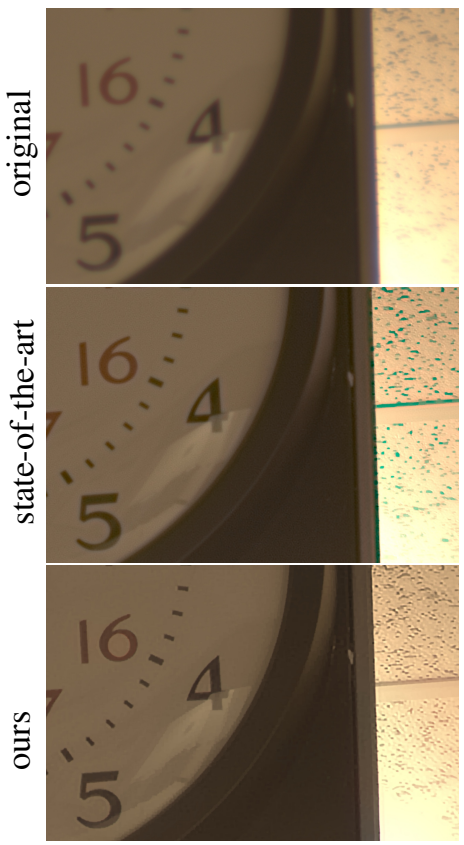

(a) Heide et al. [7]

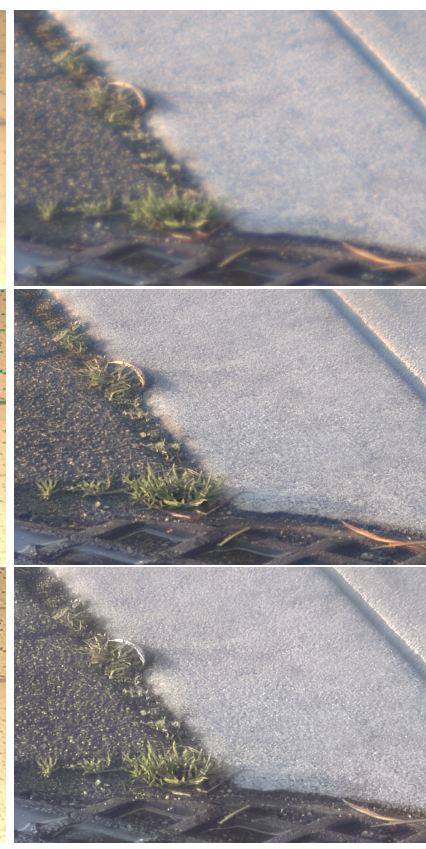

(b) Heide et al. [7]

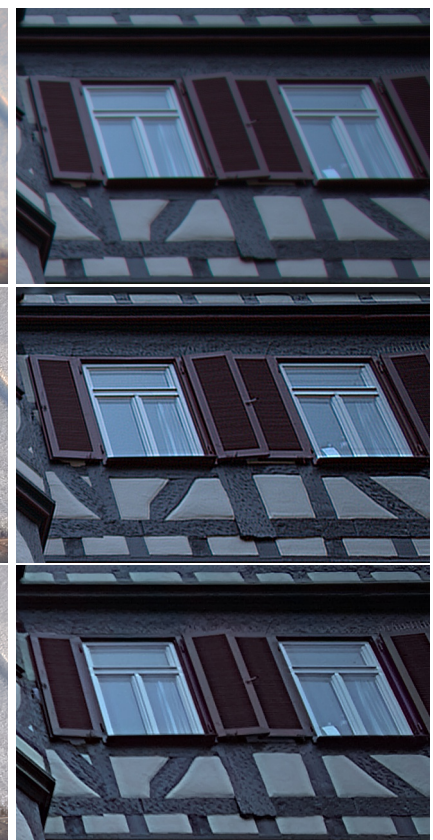

(c) Yue et al. [30]

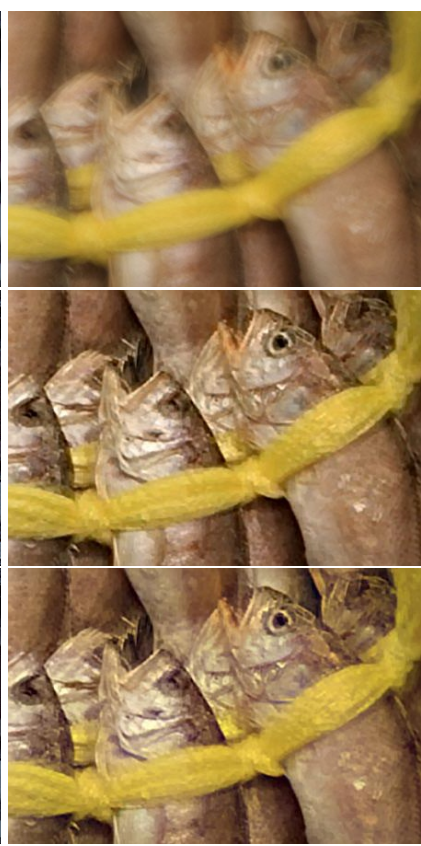

(d) Krishnan et al. [11]

Figure 9: Restored images captured by refractive lenses from state-of-the-art works. Zoom in for details.
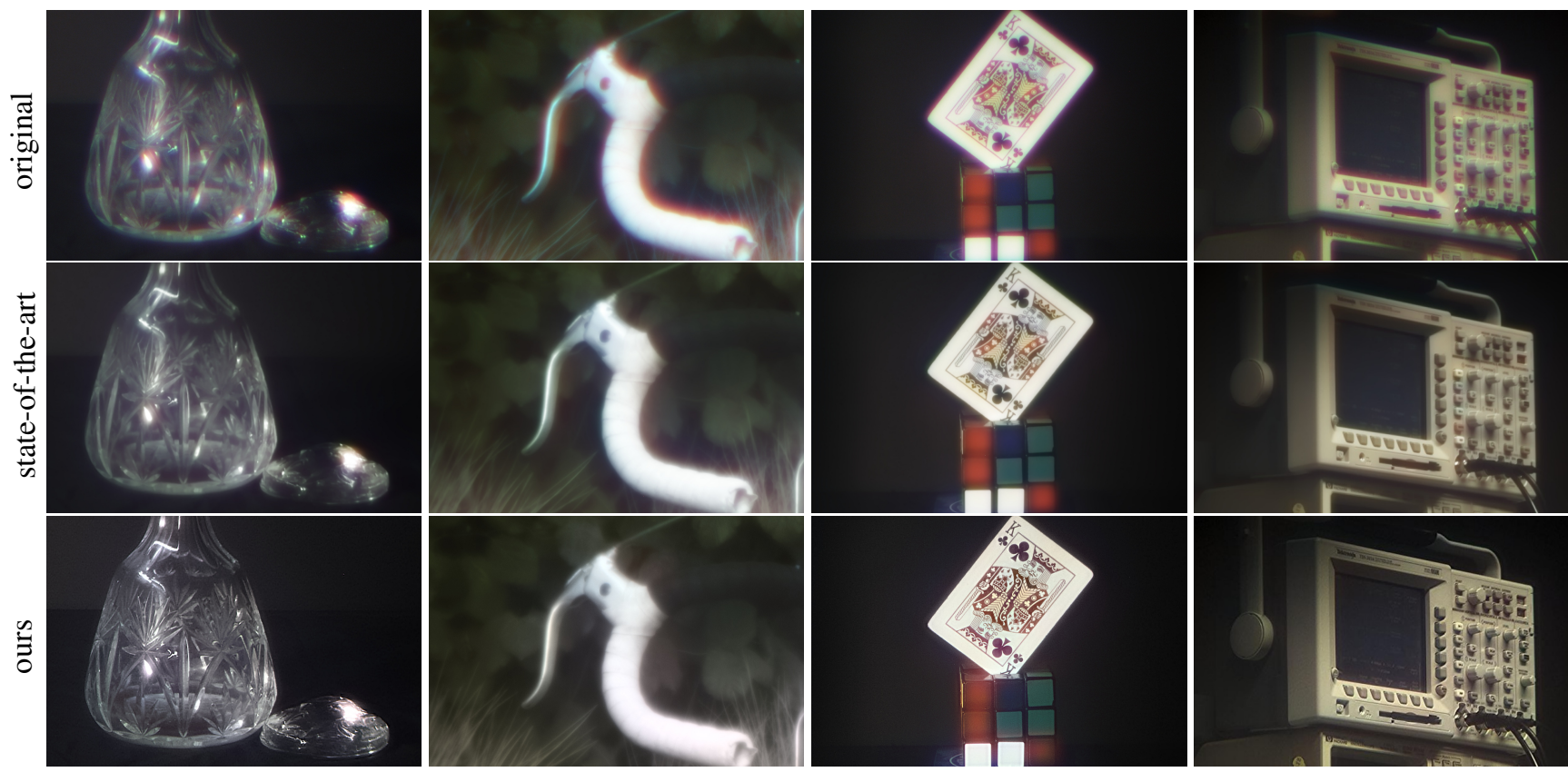

Figure 10: Restored images captured by a diffractive lens, suffering from severe chromatic aberration, with state-of-the-art reconstruction [6]. Zoom in for details.

\section{Acknowledgement}

This work was supported by KAUST baseline funding, as well as a UBC 4YF Doctoral Fellowship. The authors thank Tao Yue, Qiang Fu, and Felix Heide for the help on synthetic results.

\section{References}

[1] A. Chakrabarti and T. Zickler. Statistics of Real-World Hyperspectral Images. In Proc. IEEE Conf. on Computer Vision and Pattern Recognition (CVPR), pages 193-200, 2011.

[2] S. H. Chan, R. Khoshabeh, K. B. Gibson, P. E. Gill, and T. Q. Nguyen. An augmented lagrangian method for total variation video restoration. Image Processing, IEEE Transactions on, 
20(11):3097-3111, 2011.

[3] T. S. Cho, C. L. Zitnick, N. Joshi, S. B. Kang, R. Szeliski, and $\mathrm{W}$. T. Freeman. Image restoration by matching gradient distributions. IEEE Transactions on Pattern analysis and machine intelligence, 34(4):683-694, 2012.

[4] S.-W. Chung, B.-K. Kim, and W.-J. Song. Detecting and eliminating chromatic aberration in digital images. In Image Processing (ICIP), 2009 16th IEEE International Conference on, pages 3905-3908. IEEE, 2009.

[5] R. E. Fischer, B. Tadic-Galeb, P. R. Yoder, and R. Galeb. Optical system design. Citeseer, 2000.

[6] F. Heide, Q. Fu, Y. Peng, and W. Heidrich. Encoded diffractive optics for full-spectrum computational imaging. Scientific Reports, 6, 2016.

[7] F. Heide, M. Rouf, M. B. Hullin, B. Labitzke, W. Heidrich, and A. Kolb. High-quality computational imaging through simple lenses. ACM Transactions on Graphics (TOG), 32(5):149, 2013.

[8] B. K. Horn and B. G. Schunck. Determining optical flow. Artificial intelligence, 17(1-3):185-203, 1981.

[9] S. B. Kang. Automatic removal of chromatic aberration from a single image. In Computer Vision and Pattern Recognition, 2007. CVPR'07. IEEE Conference on, pages 1-8. IEEE, 2007.

[10] D. Krishnan and R. Fergus. Fast image deconvolution using hyper-laplacian priors. In Advances in Neural Information Processing Systems, pages 1033-1041, 2009.

[11] D. Krishnan, T. Tay, and R. Fergus. Blind deconvolution using a normalized sparsity measure. In Computer Vision and Pattern Recognition (CVPR), 2011 IEEE Conference on, pages 233-240. IEEE, 2011.

[12] A. Levin, R. Fergus, F. Durand, and W. T. Freeman. Image and depth from a conventional camera with a coded aperture. ACM transactions on graphics (TOG), 26(3):70, 2007.

[13] A. Levin, Y. Weiss, F. Durand, and W. T. Freeman. Understanding and evaluating blind deconvolution algorithms. In Computer Vision and Pattern Recognition, 2009. CVPR 2009. IEEE Conference on, pages 1964-1971. IEEE, 2009.

[14] Y. Liu, D. Zhang, G. Lu, and W.-Y. Ma. A survey of contentbased image retrieval with high-level semantics. Pattern recognition, 40(1):262-282, 2007.

[15] L. B. Lucy. An iterative technique for the rectification of observed distributions. The astronomical journal, 79:745, 1974.

[16] V. N. Mahajan and V. N. Mahajan. Aberration theory made simple. SPIE optical engineering press Bellingham, WA, 1991.

[17] K. Marwah, G. Wetzstein, Y. Bando, and R. Raskar. Compressive Light Field Photography using Overcomplete Dictionaries and Optimized Projections. ACM Trans. Graph. (Proc. SIGGRAPH), 32(4):1-11, 2013.

[18] Y. Peng, Q. Fu, H. Amata, S. Su, F. Heide, and W. Heidrich. Computational imaging using lightweight diffractiverefractive optics. Optics express, 23(24):31393-31407, 2015.

[19] Y. Peng, Q. Fu, F. Heide, and W. Heidrich. The diffractive achromat full spectrum computational imaging with diffractive optics.
[20] D. Perrone and P. Favaro. Total variation blind deconvolution: The devil is in the details. In Proceedings of the IEEE Conference on Computer Vision and Pattern Recognition, pages 2909-2916, 2014.

[21] C. J. Schuler, H. Christopher Burger, S. Harmeling, and B. Scholkopf. A machine learning approach for non-blind image deconvolution. In The IEEE Conference on Computer Vision and Pattern Recognition (CVPR), June 2013.

[22] C. J. Schuler, M. Hirsch, S. Harmeling, and B. Schölkopf. Non-stationary correction of optical aberrations. In Computer Vision (ICCV), 2011 IEEE International Conference on, pages 659-666. IEEE, 2011.

[23] C. J. Schuler, M. Hirsch, S. Harmeling, and B. Schölkopf. Blind correction of optical aberrations. In European Conference on Computer Vision, pages 187-200. Springer, 2012.

[24] Q. Shan, J. Jia, and A. Agarwala. High-quality motion deblurring from a single image. In ACM Transactions on Graphics (TOG), volume 27, page 73. ACM, 2008.

[25] D. G. Stork and P. R. Gill. Optical, mathematical, and computational foundations of lensless ultra-miniature diffractive imagers and sensors. International Journal on Advances in Systems and Measurements, 7(3):4, 2014.

[26] L. Sun, S. Cho, J. Wang, and J. Hays. Edge-based blur kernel estimation using patch priors. In Computational Photography (ICCP), 2013 IEEE International Conference on, pages 1-8. IEEE, 2013.

[27] P. Wang, N. Mohammad, and R. Menon. Chromaticaberration-corrected diffractive lenses for ultra-broadband focusing. Scientific reports, 6, 2016.

[28] Z. Wang, A. C. Bovik, H. R. Sheikh, and E. P. Simoncelli. Image quality assessment: from error visibility to structural similarity. IEEE transactions on image processing, 13(4):600-612, 2004.

[29] N. Wiener. Extrapolation, Interpolation, and Smoothing of Stationary Time Series. The MIT Press, 1964.

[30] T. Yue, J. Suo, J. Wang, X. Cao, and Q. Dai. Blind optical aberration correction by exploring geometric and visual priors. In The IEEE Conference on Computer Vision and Pattern Recognition (CVPR), June 2015.

[31] T. Yue, J. Suo, Y. Xiao, L. Zhang, and Q. Dai. Image quality enhancement using original lens via optical computing. Optics express, 22(24):29515-29530, 2014. 